\title{
repisälud
}

This is the peer reviewed version of the following article:

Dorado B, Andres V. A-type lamins and cardiovascular disease in premature aging syndromes. Curr Opin Cell Biol. 2017;46:17-25

which has been published in final form at: https://doi.org/10.1016/i.ceb.2016.12.005 


\section{A-type lamins and cardiovascular disease in premature aging syndromes}

Beatriz Dorado and Vicente Andrés

Centro Nacional de Investigaciones Cardiovasculares Carlos III (CNIC), Melchor Fernández Almagro 3, 28029, Madrid, Spain

Corresponding Author: V. Andrés

e-mail addresses

V. Andrés: vandres@cnic.es

B. Dorado: bjdorado@cnic.es

SHORT TITLE: A-type lamins and cardiovascular disease in progeria

\section{HIGHLIGHTS:}

- A-type lamins ( $L M N A$ gene) play important structural and regulatory roles.

- Multiple diseases arise from $L M N A$ mutations, including progeroid syndromes.

- Premature death in progeria is caused by severe cardiovascular disease.

- Diverse mechanisms perturb cellular and whole-body homeostasis in progeria.

- Additional progeria-causing mechanisms remain unknown. 


\section{ABSTRACT}

Lamin A is a nuclear intermediate filament protein with important structural and regulatory roles in most differentiated mammalian cells. Excessive accumulation of its precursor prelamin A or the mutant form called 'progerin' causes premature aging syndromes. Progeroid 'laminopathies' are characterized by severe cardiovascular problems (cardiac electrical defects, vascular calcification and stiffening, atherosclerosis, myocardial infarction, and stroke) and premature death. Here, we review studies in cell and mouse models and patients that are unraveling how abnormal prelamin $\mathrm{A}$ and progerin accumulation accelerates cardiovascular disease and aging. This knowledge is essential for developing effective therapies to treat progeria and may help identify new mechanisms underlying normal aging.

\section{INTRODUCTION}

A-type lamins (Lamin A and C, encoded by the LMNA gene in human chromosome 1, National Centre for Biotechnology Information Reference Sequence NG_008692.2) are key components of the nuclear envelope [1]. Mature lamin A is generated through sequential posttranslational modifications of the precursor protein prelamin $\mathrm{A}$, which normally does not accumulate in cells (Figure 1A). Maturation processing of prelamin A involves the following steps: 1) farnesylation at the cysteine residue of the C-terminal cysteine-serine-isoleucine-methionine motif (CSIM), 2) cleavage of the terminal SIM residues, 3) carboxymethylation of the farnesylcysteine, and 4) endoproteolytic removal of the 15 C-terminal aminoacids - including the farnesylcysteine $\alpha$-methyl ester-by the zinc metalloprotease ZMPSTE24/FACE-1 [2]. The first three modifications render the protein more hydrophobic and facilitate its interaction with the nuclear membrane, and cleavage of the farnesyl-group increases the flexibility of lamin A once integrated in the nuclear lamina [3].

A-type lamins are expressed in most differentiated mammalian cells [1]. The lamin field has traditionally focused on the roles played by these filamentous proteins in maintaining nuclear architecture. However, groundbreaking work over the last few years has demonstrated that A-type lamins and associated nuclear envelope proteins also regulate multiple cell functions, including DNA replication and repair, higher-order chromatin organization, signal transduction, and gene transcription [4,5]. Interest in A-type lamins has acquired added relevance with the discovery that $L M N A$ mutations, or other genetic defects leading to changes in lamin A abundance or post-translational processing, cause at least 12 human disorders termed laminopathies [6].

Most laminopathy symptoms develop during childhood or adolescence, but some laminopathies are lethal at very young age, such as those affecting individuals with ZMPSTE24 mutations causing abnormal accumulation of farnesylated prelamin A [7] and Hutchinson-Gilford progeria syndrome (HGPS) patients, who express a mutant form of prelamin A called progerin [2,8] (Figure 1B). HGPS has an estimated prevalence of 1 in 20 million (www.progeriaresearch.org). Most HGPS patients carry in heterozygosis a de novo dominant synonymous $L M N A$ mutation (c.1824C $>$ T: GGC $>$ GGT; p.G608G) which activates an aberrant 5 ' splicing site in exon $11[9,10]$. Abnormal splicing leads to the 
synthesis of progerin, which lacks 50 aminoacid residues encompassing the ZMPSTE24 cleavage site. Like prelamin A, progerin retains the farnesylated modification at the Cterminus and remains permanently attached to the nuclear membrane. HGPS is characterized by severe failure to thrive, alopecia, joint contractures, scleroderma-like skin, lipodystrophy, and skeletal dysplasia $\left[8,11,12^{* *}, 13\right]$. HGPS patients also develop exacerbated cardiovascular disease (CVD), including cardiac electrical defects, atherosclerosis, vascular stiffening and calcification, and die at an average age of 14.6 years, predominantly from myocardial infarction or stroke $\left[8,11,12^{* *}, 13,14^{* *}\right]$ (Figure 2). Abnormal prelamin A accumulation due to ZMPSTE24 mutations causes progeroid syndromes that share key features with HGPS, including premature aging [7].

Cell and animal models are essential for understanding the molecular mechanisms causing progeria and for the identification of therapeutic targets [15-17]. Preclinical studies have shown that the progeroid phenotype can be ameliorated by treatment with farnesyltransferase inhibitors (FTIs) [17,18], prompting several recent single-arm clinical trials of lonafarnib to reduce progerin toxicity. This FTI improved some aspects of cardiovascular and bone disease and audiological status in patient subgroups [19], and Kaplan-Meier survival analysis suggested increased mean survival by 1.6 years in treated patients [12]. The low efficiency of FTIs in ameliorating progeria symptoms may be due to alternative prenylation by geranylgeranyltransferase in the setting of farnesyltransferase inhibition [20]. Supporting this view, the longevity of progeroid Zmpste24-null mice is substantially extended by combined treatment with statins and aminobisphosphonates to simultaneously inhibit progerin and prelamin A farnesylation and geranylgeranylation [20]. This finding has been followed up in a 'triple-drug' clinical trial to evaluate the efficacy of combination therapy with an FTI (lonafarnib), a statin (pravastatin), and an aminobisphosphonate (zoledronic acid) [21**]. No participants withdrew because of side effects, and comparisons with lonafarnib monotherapy revealed an additional bone mineral density benefit; however, some patients showed increased rates of carotid and femoral arterial plaques and extraskeletal calcification, and addition of pravastatin and zoledronic acid produced no added cardiovascular benefit.

Further research is clearly needed to improve HGPS therapies and to find a cure for this devastating disease. Since CVD is the main cause of death in HGPS, here we review the molecular mechanisms by which prelamin A and progerin cause cardiovascular damage. This knowledge may shed light on the molecular mechanisms driving physiological aging and associated CVD, since progerin and prelamin A are both expressed in cells and tissues of normally aging non-HGPS individuals $[2,8]$.

\section{VASCULAR DISEASE IN PROGERIA}

\section{Vascular smooth muscle cell loss}

Alterations in vascular smooth muscle cells (VSMCs) play a major role in the development of vascular disease associated with normal and premature aging. HGPS patients exhibit VSMC loss with accumulation of matrix proteoglycans in the aorta and carotid arteries [22,23], and similar changes have been reported in arteries from progerinexpressing mice [24,25]. Suppression of poly (ADP-ribose) polymerase 1 (PARP1) in human VSMCs differentiated from induced pluripotent stem cells of HGPS patients activates error-prone non-homologous end joining of DNA double-strand breaks, prolonging mitosis and causing mitotic catastrophe and caspase-independent cell death 
[26*]. Future studies are required to identify additional mechanisms causing VSMC loss in HGPS, and to assess the contribution of VSMC death to progerin-induced vascular disease.

\section{Vascular calcification}

Vascular calcification (VC) increases with ageing and contributes to CVD-associated morbimortality [27]. Excessive VC occurs both in HGPS patients (aortic calcification, calcific aortic valve stenosis, and calcific mitral valve) [28-30] and HGPS-like mouse models (aortic calcification) [24,31]. VC is regulated by a tight balance between activators and inhibitors, many of which are produced by VSMCs. Extracellular inorganic pyrophosphate (ePPi) is a major inhibitor of calcification $[32,33]$, and three lines of evidence indicate that defective ePPi metabolism promotes $\mathrm{VC}$ in progerin-expressing $\mathrm{Lmna}^{\mathrm{G} 609 \mathrm{G} / \mathrm{+}}$ mice [31] (Figure 3). First, primary VSMCs derived from $\mathrm{Lmna}^{\mathrm{G} 609 \mathrm{G} / \mathrm{+}}$ aorta display mitochondrial dysfunction, leading to reduced synthesis of ATP (the main substrate for ePPi synthesis), as well as upregulation of the ectoenzymes tissuenonspecific alkaline phosphatase (TNAP, the main enzyme involved in ePPi hydrolysis) and apyrase1/eNTPD1 (which hydrolizes ATP to release inorganic phosphate). These alterations cause defective production and accumulation of ePPi. Second, Lmna ${ }^{G 609 G /+}$ mice have higher alkaline phosphatase activity and lower ATP and PPi levels in plasma. Finally, PPi treatment inhibits VC in $L m n a^{G 609 G /+}$ mice. Future studies are thus warranted to elucidate the molecular mechanisms causing progerin-dependent TNAP and apyrase1/eNTPD1 upregulation and mitochondrial dysfunction in VSMCs, and to investigate whether restoring PPi levels can attenuate VC in HGPS.

Recent studies have identified prelamin A as a biomarker and driver of human vascular aging that accelerates VSMC senescence and calcification at least in part by promoting the accumulation of DNA damage and the expression of osteogenic markers (Figure 4). Oxidative stress reduces endogenous ZMPSTE24/FACE1 expression in VSMCs, and prelamin A accumulation occurs in VSMCs undergoing aging in vitro, in medial VSMCs from aged individuals, and in atherosclerotic lesions, where it often colocalizes with senescent and degenerate VSMCs [34]. In cultured VSMCs, ZMPSTE24/FACE1 silencing, treatment with HIV-protease inhibitors (both leading to increased expression of endogenous prelamin A), and prelamin A overexpression all promote inflammation, senescence, and calcification [34,35]. Prelamin A accumulation in VSMCs triggers a persistent amplification of the DNA damage response (DDR) directed by increased activity of ataxia-telangiectasia-mutated (ATM)- and ataxia-telangiectasia Rad3 (ATR)related kinases [36]. As a consequence, prelamin A-positive VSMCs release the calcification modulators interleukin 6, bone morphogenic protein 2 and osteoprotegerin, along with other factors and cytokines characteristic of the senescence-associated secretory phenotype [36] (Figure 4). Prelamin A expression in VSMCs also induces expression of the calcification regulators osteocalcin and osteopontin, as well as expression and nuclear translocation of osteoblast-specific Run-related transcription factor-2 (RUNX2) [37]. Increased DDR and prelamin A levels induce, via nesprin-2 and lamin A interactions, the segregation of extracellular signal-regulated kinases 1 and 2 (ERK1/2) to promyelocytic leukaemia protein nuclear bodies at sites of DNA damage [38]. Prelamin A may also impair DDR in aged VSMCs through a defective nuclear import of 53 binding protein-1 (53BP1), associated with a deregulated Ran gradient causing abnormal topological arrangement of nucleoporin NUP153 [39*]. Treatment with 
remodelin, an inhibitor of the N-acetyl-transferase NAT10 that improves the phenotype of HGPS fibroblasts [40**], alleviates deficiencies associated with nuclear lamina alterations in VSMCs, including NUP153 disorganization, 53BP1 cytoplasmic accumulation, and senescence [39]; however the underlying molecular mechanism remains elusive.

\section{Atherosclerosis}

Atherosclerosis and vascular stiffening are highly prevalent in HGPS patients and in the geriatric population $[12 * *, 19,23,27,41,42]$. Although many similarities have been found between atherosclerotic plaques from normally aged individuals and HGPS patients, HGPS lesions exhibit more prominent fibrosis and adventitial thickening $[11,23]$. In HGPS patients, progerin is expressed in the nuclei of a very high proportion of medial VSMCs, neointimal cells, adventitial fibroblasts, and arteriolar VSMCs and endothelial cells (ECs). Interestingly, progerin is detected at a very low rate in the cell cytoplasm in coronary arteries in non-HGPS individuals, and its expression increases significantly with aging (mean progerin staining rate at the age of 97 years: $\sim 20$ cells per 1000 cells in adventitia and $\sim 1$ cell per 1000 cells in media and plaque) [23].

Atherosclerosis occurs mainly in arterial segments with curvatures and branching, which display EC dysfunction triggered by low shear stress (SS) or oscillatory and turbulent SS $[43,44]$. Evidence is accumulating that A-type lamins and associated NE proteins are key regulators of "outside-in" and "inside-out" signaling, and that mechanotransduction functions may be impaired by prelamin A and progerin, especially in mechanicallystressed tissues [45]. Compared with normal SS, low SS suppresses EC expression of nesprin2 and lamin $\mathrm{A}$, and this suppression might subsequently modulate the activation of important transcription factors, leading to EC dysfunction and subendothelial migration of blood-borne immune cells [46]. This view is supported by the finding that siRNA-mediated reduction of lamin $\mathrm{A} / \mathrm{C}$ expression in ECs facilitates subendothelial migration of T cells, possibly due to the presence of a less stiff EC layer [47*].

Song et al. [48] investigated the effects of SS on the expression of mechanotransduction proteins in the aorta of $m L M N A+$ mice, a progeroid transgenic mouse model carrying a human bacterial artificial chromosome that harbors the common c.1824C $>\mathrm{T}$ (G608G) HGPS mutation [24]. In these progerin-expressing mice, medial VSMCs of the ascending aorta subjected to high SS showed signs of vascular disease correlating with dysregulation of mechanotransduction proteins, including vimentin, which was not observed in descending aorta [48]. Importantly, SS applied ex vivo to the descending aorta of $m L M N A+$ mice caused a significant reduction in vimentin mRNA and protein levels but did not affect its expression in wild-type tissue. Increased sensitivity of progerinexpressing VSMCs to pro-atherogenic SS may therefore contribute to vascular disease in progeria. It will be interesting to investigate whether prelamin A and progerin exacerbate SS-mediated EC dysfunction and subendothelial migration of circulating immune cells, as well as other pro-atherogenic functions of immune cells.

In differentiated ECs, the anti-retroviral Atazanavir induces prelamin A accumulation and promotes intercellular adhesion molecule 1 (ICAM1)-dependent monocyte adhesion to ECs [49]. In line with this observation, NF-kB activation and secretion of high levels of pro-inflammatory cytokines occur in prelamin A-expressing Zmpste24 $4^{-/}$and progerin- 
expressing $\mathrm{Lmna}^{\mathrm{G} 609 \mathrm{G} / \mathrm{G} 609 \mathrm{G}}$ mice, and NF-kB inhibition prevents age-associated features and extended longevity in these progeroid models [50]. In addition, alterations in the many nuclear functions modulated by lamin A may contribute to vascular cell senescence and dysfunction. For example, accumulation of prelamin A isoforms causes genomic instability through diverse mechanisms, including telomere dysfunction [51], increased oxidative stress and DDR [39,52], abnormal chromosome segregation during mitosis [53*], epigenetic changes [54,55], and dysregulated gene transcription [4,56]. Prelamin A isoforms can also inhibit vascular repair by inducing senescence of stem cells and their progenitors [16,41]. Remarkably, repression of the antioxidant NRF2 pathway has been proposed as a driver of HGPS, with NRF2 reactivation decreasing oxidative stress and alleviating HGPS mesenchymal stem cell viability defects in an animal model [57**]. Mitochondrial dysfunction may also contribute to progerin-induced oxidative stress [58].

\section{CARDIAC ELECTRICAL DEFECTS IN PROGERIA}

Myocardial infarction and stroke resulting from widespread atherosclerosis are considered the main causes of death in HGPS [8,11,12**,13]. Several studies have also demonstrated cardiac electrical defects in HGPS patients and progeroid mice. Merideth et al. performed a thorough clinical evaluation of 15 HGPS patients aged between 1 and 17 years [11]. Electrocardiographic testing showed long QT intervals in five children, including the three oldest. We recently confirmed and extended these findings by analyzing another cohort of 15 HGPS patients aged between 2 and 19 years [14**]. Half of the patients showed overt repolarization abnormalities in at least one electrocardiogram, and these abnormalities were highly evident at advanced disease stages. HGPS patients also exhibited significant T-wave flattening, which was exacerbated with age. Although heart rate and PR interval were within the physiological range for all HGPS patients, heart rate in older patients tended to be slower and PR interval larger. Studies in progerin-expressing $\mathrm{Lmma}^{\mathrm{G} 609 \mathrm{G} / G 609 G}$ mice [25] and prelamin A-expressing Zmpste24/- mice [14**] revealed fibrosis-unrelated bradycardia as well as PQ interval and QRS complex prolongation with age. In addition, patch-clamping in Zmpste $24^{-/}$cardiomyocytes demonstrated prolonged duration of calcium transients and reduced sarcoplasmic reticulum calcium loading capacity and release, consistent with the absence of isoproterenol-induced ventricular arrhythmias in Zmpste $24^{-/}$mice. Conduction defects in HGPS patients and Zmpste $24^{-/}$mice are accompanied by overt mislocalization of connexin 43 [14**], a gap-junction protein which is essential for proper intercellular electrical coupling between cardiomyocytes and for action potential spread during each cardiac cycle.

Collectively, these studies suggest that cardiac electrical defects associated with impaired cardiomyocyte connectivity might cause cardiac rhythm alterations and premature death in HGPS. Future studies are warranted to establish direct causal connections between prelamin A or progerin accumulation and cardiac abnormalities in HGPS, and to elucidate the underlying mechanisms (e.g., how these proteins cause connexin 43 mislocalization). Such studies may pave the way to new therapies for HGPS patients.

\section{CONCLUDING REMARKS}

A-type lamins regulate nuclear architecture and multiple cell functions, including DNA replication and repair, higher-order chromatin organization, signal transduction, and gene transcription. Interest in A-type lamins has increased with the discovery that abnormal 
accumulation of prelamin A or its mutant form progerin cause progeroid syndromes characterized by excessive CVD and premature death. Despite major progress in the last decade, future studies are warranted to continue elucidating the functional consequences of the many interactions that prelamin A and progerin maintain with other ubiquitous or tissue-specific proteins, and how they promote atherosclerosis, calcification, and cardiac electrical defects. There are exciting prospects for high-throughput technologies in combination with functional studies targeting candidate factors and the generation of new conditional or tissue-specific small and large animal progeria models. These studies will help to expedite the identification of new targets and the translation of basic knowledge into effective therapies for HGPS. Because non-HGPS individuals express low levels of prelamin A and progerin in aging cells and tissues, including cardiovascular tissues, efforts to find a cure for premature aging syndromes might also help to promote healthy aging in the general population.

\section{ACKNOWLEDGEMENTS}

We thank M. J. Andrés-Manzano for help with preparation of the figures and Simon Bartlett for English editing. Work in the laboratory of V.A. is supported by the Spanish Ministerio de Economía, Industria y Competitividad (MINECO) (SAF2013-46663-R) and the Instituto de Salud Carlos III (RD12/0042/0028) with co-funding from the Fondo Europeo de Desarrollo Regional (FEDER), the Fundació Marató TV3 (122/C/2015), and the Progeria Research Foundation (Established Investigator Award 2014-52). The CNIC is supported by the MINECO and the Pro-CNIC Foundation, and is a Severo Ochoa Center of Excellence (MINECO award SEV-2015-0505). The authors declare that they have no conflicting financial interests. 


\section{REFERENCES AND RECOMMENDED READING}

Papers of particular interest, published within the period of review, have been highlighted as:

* of special interest

** of outstanding interest

1. Dittmer TA, Misteli T: The lamin protein family. Genome Biol 2011, 12:222.

2. Trigueros-Motos L, González JM, Rivera J, Andrés V: Hutchinson-Gilford progeria syndrome, cardiovascular disease and oxidative stress. Front Biosc 2011, 3:12851297.

3. Casasola A, Scalzo D, Nandakumar V, Halow J, Recillas-Targa F, Groudine M, Rincon-Arano $\mathrm{H}$ : Prelamin A processing, accumulation and distribution in normal cells and laminopathy disorders. Nucleus 2016, 7:84-102.

4. Andrés V, González JM: Role of A-type lamins in signaling, transcription, and chromatin organization. J Cell Biol 2009, 187:945-957.

5. Gruenbaum Y, Foisner R: Lamins: nuclear intermediate filament proteins with fundamental functions in nuclear mechanics and genome regulation. Annu Rev Biochem 2015, 84:131-164.

6. Worman HJ: Nuclear lamins and laminopathies. J Pathol 2012, 226:316-325.

7. Barrowman J, Wiley PA, Hudon-Miller SE, Hrycyna CA, Michaelis S: Human ZMPSTE24 disease mutations: residual proteolytic activity correlates with disease severity. Hum Mol Genet 2012, 21:4084-4093.

8. Gordon LB, Rothman FG, Lopez-Otin C, Misteli T: Progeria: a paradigm for translational medicine. Cell 2014, 156:400-407.

9. De Sandre-Giovannoli A, Bernard R, Cau P, Navarro C, Amiel J, Boccaccio I, Lyonnet S, Stewart CL, Munnich A, Le Merrer M, et al.: Lamin a truncation in HutchinsonGilford progeria. Science 2003, 300:2055.

10. Eriksson M, Brown WT, Gordon LB, Glynn MW, Singer J, Scott L, Erdos MR, Robbins CM, Moses TY, Berglund $\mathrm{P}$, et al.: Recurrent de novo point mutations in lamin A cause Hutchinson-Gilford progeria syndrome. Nature 2003, 423:293298.

11. Merideth MA, Gordon LB, Clauss S, Sachdev V, Smith AC, Perry MB, Brewer CC, Zalewski C, Kim HJ, Solomon B, et al.: Phenotype and course of HutchinsonGilford progeria syndrome. $N$ Engl J Med 2008, 358:592-604.

** 12. Gordon LB, Massaro J, D'Agostino RB, Sr., Campbell SE, Brazier J, Brown WT, Kleinman ME, Kieran MW, Progeria Clinical Trials C: Impact of farnesylation inhibitors on survival in Hutchinson-Gilford progeria syndrome. Circulation 2014, 130:27-34.

The authors estimated a mean survival of 14.6 years for untreated HGPS patients. An increase in mean survival by 1.6 years was estimated in patients treated with the FTI lonafarnib alone or combined with zoledronate (aminobisphosphonate) and 
pravastatin (statin). This study provides the first evidence of FTI therapy influencing survival for HGPS.

13. Ullrich NJ, Gordon LB: Hutchinson-Gilford progeria syndrome. Handb Clin Neurol 2015, 132:249-264.

**14. Rivera-Torres J, Calvo CJ, Llach A, Guzmán-Martínez G, Caballero R, GonzálezGómez C, Jiménez-Borreguero LJ, Guadix JA, Osorio FG, López-Otín C, et al.: Cardiac electrical defects in progeroid mice and Hutchinson-Gilford progeria syndrome patients with nuclear lamina alterations. Proc. Natl. Acad. Sci. U.S.A. 2016, 113:E7250-E7259.

Age-dependent cardiac repolarization abnormalities develop in both HGPS patients and progeroid Zmpste $24^{-/}$mice associated with mislocalization of connexin 43 in the heart. Since connexin 43 is essential for proper intercellular electrical coupling between cardiomyocytes and for action potential spread during each cardiac cycle, these results reveal alterations that might cause cardiac rhythm alterations and premature death in HGPS.

15. Gonzalo S, Kreienkamp R, Askjaer P: Hutchinson-Gilford Progeria Syndrome: A premature aging disease caused by LMNA gene mutations. Ageing Res Rev 2016.

16. Vidak S, Foisner R: Molecular insights into the premature aging disease progeria. Histochem Cell Biol 2016, 145:401-417.

17. Zhang H, Kieckhaefer JE, Cao K: Mouse models of laminopathies. Aging cell 2013, 12:2-10.

18. Osorio FG, Obaya AJ, López-Otín C, Freije JM: Accelerated ageing: from mechanism to therapy through animal models. Transgenic Res 2009, 18:7-15.

19. Gordon LB, Kleinman ME, Miller DT, Neuberg DS, Giobbie-Hurder A, GerhardHerman M, Smoot LB, Gordon CM, Cleveland R, Snyder BD, et al.: Clinical trial of a farnesyltransferase inhibitor in children with Hutchinson-Gilford progeria syndrome. Proc Natl Acad Sci U S A 2012, 109:16666-16671.

20. Varela I, Pereira S, Ugalde AP, Navarro CL, Suárez MF, Cau P, Cadiñanos J, Osorio FG, Foray N, Cobo J, et al.: Combined treatment with statins and aminobisphosphonates extends longevity in a mouse model of human premature aging. Nat Med 2008, 14:767-772.

** 21. Gordon LB, Kleinman ME, Massaro J, D'Agostino RB, Sr., Shappell H, GerhardHerman M, Smoot LB, Gordon CM, Cleveland RH, Nazarian A, et al.: Clinical trial of the protein farnesylation inhibitors lonafarnib, pravastatin, and zoledronic acid in children with Hutchinson-Gilford progeria syndrome. Circulation 2016, 134:114-125.

Gordon et al. report the results of a 'triple-drug' clinical trial designed to evaluate in HGPS patients the efficacy of combination therapy with lonafarnib, pravastatin and zoledronate. Although some patients showed additional bone mineral density benefit, a subgroup had increased rates of carotid and femoral arterial plaques and extraskeletal calcifications. Overall, addition of pravastatin and zoledronate did not appear to add cardiovascular benefit compared with lonafarnib monotherapy. 
22. Capell BC, Collins FS, Nabel EG: Mechanisms of cardiovascular disease in accelerated aging syndromes. Circ Res 2007, 101:13-26.

23. Olive M, Harten I, Mitchell R, Beers JK, Djabali K, Cao K, Erdos MR, Blair C, Funke $\mathrm{B}$, Smoot L, et al.: Cardiovascular pathology in Hutchinson-Gilford progeria: correlation with the vascular pathology of aging. Arterioscler Thromb Vasc Biol 2010, 30:2301-2309.

24. Varga R, Eriksson M, Erdos MR, Olive M, Harten I, Kolodgie F, Capell BC, Cheng $\mathrm{J}$, Faddah D, Perkins S, et al.: Progressive vascular smooth muscle cell defects in a mouse model of Hutchinson-Gilford progeria syndrome. Proc Natl Acad Sci U $S$ A 2006, 103:3250-3255.

25. Osorio FG, Navarro CL, Cadiñanos J, López-Mejía IC, Quirós PM, Bartoli C, Rivera J, Tazi J, Guzmán G, Varela I, et al.: Splicing-directed therapy in a new mouse model of human accelerated aging. Sci Trans Med 2011, 3:106ra107.

* 26. Zhang H, Xiong ZM, Cao K: Mechanisms controlling the smooth muscle cell death in progeria via down-regulation of poly(ADP-ribose) polymerase 1. Proc Natl Acad Sci U S A 2014, 111:E2261-2270.

The authors generated VSMCs derived from HGPS induced pluripotent stem cells to unravel mechanisms leading to VSMC loss. Downregulation and mislocalization of Poly(ADP-ribose) polymerase 1 (PARP1), and activation of the error-prone nonhomologous end joining response, are proposed to link progerin-induced defective DNA damage repair and VSMC death.

27. Kovacic JC, Moreno P, Nabel EG, Hachinski V, Fuster V: Cellular senescence, vascular disease, and aging: part 2 of a 2-part review: clinical vascular disease in the elderly. Circulation 2011, 123:1900-1910.

28. Hanumanthappa NB, Madhusudan G, Mahimarangaiah J, Manjunath CN: Hutchinson-Gilford progeria syndrome with severe calcific aortic valve stenosis. Ann Pediatr Cardiol 2011, 4:204-206.

29. Nair K, Ramachandran P, Krishnamoorthy KM, Dora S, Achuthan TJ: HutchinsonGilford progeria syndrome with severe calcific aortic valve stenosis and calcific mitral valve. J Heart Valve Dis 2004, 13:866-869.

30. Salamat M, Dhar PK, Neagu DL, Lyon JB: Aortic calcification in a patient with Hutchinson-Gilford progeria syndrome. Pediatr Cardiol 2010, 31:925-926.

31. Villa-Bellosta R, Rivera-Torres J, Osorio FG, Acín-Pérez R, Enríquez JA, López-Otín C, Andrés V: Defective extracellular pyrophosphate metabolism promotes vascular calcification in a mouse model of Hutchinson-Gilford progeria syndrome that is ameliorated on pyrophosphate treatment. Circulation 2013, 127:2442-2451.

32. Villa-Bellosta R, Wang X, Millan JL, Dubyak GR, O'Neill WC: Extracellular pyrophosphate metabolism and calcification in vascular smooth muscle. $A m J$ Physiol Heart Circ Physiol 2011, 301:H61-68.

33. Villa-Bellosta $\mathrm{R}$, Sorribas V: Prevention of vascular calcification by polyphosphates and nucleotides- role of ATP. Circ. J 2013, 77:2145-2151. 
34. Ragnauth CD, Warren DT, Liu Y, McNair R, Tajsic T, Figg N, Shroff R, Skepper J, Shanahan CM: Prelamin A acts to accelerate smooth muscle cell senescence and is a novel biomarker of human vascular aging. Circulation 2010, 121:2200-2210.

35. Afonso P, Auclair M, Boccara F, Vantyghem MC, Katlama C, Capeau J, Vigouroux C, Caron-Debarle M: LMNA mutations resulting in lipodystrophy and HIV protease inhibitors trigger vascular smooth muscle cell senescence and calcification: Role of ZMPSTE24 downregulation. Atherosclerosis 2016, 245:200-211.

36. Liu Y, Drozdov I, Shroff R, Beltran LE, Shanahan CM: Prelamin A accelerates vascular calcification via activation of the DNA damage response and senescence-associated secretory phenotype in vascular smooth muscle cells. Circ Res 2013, 112:e99-109.

37. Quirós-González I, Román-García P, Alonso-Montes C, Barrio-Vázquez S, CarrilloLópez N, Naves-Díaz M, Mora MI, Corrales FJ, López-Hernández FJ, Ruiz-Torres MP, et al.: Lamin $A$ is involved in the development of vascular calcification induced by chronic kidney failure and phosphorus load. Bone 2016, 84:160-168.

38. Warren DT, Tajsic T, Porter LJ, Minaisah RM, Cobb A, Jacob A, Rajgor D, Zhang QP, Shanahan CM: Nesprin-2-dependent ERK1/2 compartmentalisation regulates the DNA damage response in vascular smooth muscle cell ageing. Cell Death Differ 2015, 22:1540-1550.

* 39. Cobb AM, Larrieu D, Warren DT, Liu Y, Srivastava S, Smith AJ, Bowater RP, Jackson SP, Shanahan CM: Prelamin A impairs 53BP1 nuclear entry by mislocalizing NUP153 and disrupting the Ran gradient. Aging Cell 2016.

Prelamin A inhibits 53BP1 recruitment to sites of DNA damage and increases basal levels of DNA damage in aged VSMCs due to defective nuclear import of 53BP1 as a consequence of mislocalization of the nucleoporin NUP153. Many of these defects were reverted by Remodelin, an inhibitor of the N-acetyl-transferase NAT10.

** 40. Larrieu D, Britton S, Demir M, Rodriguez R, Jackson SP: Chemical inhibition of NAT10 corrects defects of laminopathic cells. Science 2014, 344:527-532.

The authors identified the small molecule "Remodelin", which decreases markers of DNA damage and improves nuclear architecture, chromatin organization, and fitness of human lamin A/C-depleted cells and HGPS-derived patient cells. The acetyltransferase protein NAT10 is identified as the target of Remodelin which mediates microtubule reorganization and nuclear shape rescue in laminopathic cells.

41. Brassard JA, Fekete N, Garnier A, Hoesli CA: Hutchinson-Gilford progeria syndrome as a model for vascular aging. Biogerontology 2016, 17:129-145.

42. Gerhard-Herman M, Smoot LB, Wake N, Kieran MW, Kleinman ME, Miller DT, Schwartzman A, Giobbie-Hurder A, Neuberg D, Gordon LB: Mechanisms of premature vascular aging in children with Hutchinson-Gilford progeria syndrome. Hypertension 2012, 59:92-97.

43. Baeyens N, Bandyopadhyay C, Coon BG, Yun S, Schwartz MA: Endothelial fluid shear stress sensing in vascular health and disease. $J$ Clin Invest 2016, 126:821828. 
44. Chiu JJ, Chien S: Effects of disturbed flow on vascular endothelium: pathophysiological basis and clinical perspectives. Physiol Rev 2011, 91:327-387.

45. Osmanagic-Myers $\mathrm{S}$, Dechat $\mathrm{T}$, Foisner $\mathrm{R}$ : Lamins at the crossroads of mechanosignaling. Genes Dev 2015, 29:225-237.

46. Han Y, Wang L, Yao QP, Zhang P, Liu B, Wang GL, Shen BR, Cheng B, Wang Y, Jiang $Z L$, et al.: Nuclear envelope proteins Nesprin2 and LaminA regulate proliferation and apoptosis of vascular endothelial cells in response to shear stress. Biochim Biophys Acta 2015, 1853:1165-1173.

* 47. Song KH, Lee J, Park H, Kim HM, Park J, Kwon KW, Doh J: Roles of endothelial A-type lamins in migration of $\mathbf{T}$ cells on and under endothelial layers. Sci Rep 2016, 6:23412.

siRNA-mediated downregulation of A-type lamins in ECs facilitates the migration of $\mathrm{T}$ cells through EC layers. This result suggests that regulation of EC nuclear stiffness by lamin $\mathrm{A} / \mathrm{C}$ may modulate subendothelial migration of blood-borne immune cells, a key process in all phases of atherosclerosis.

48. Song M, San H, Anderson SA, Cannon RO, 3rd, Orlic D: Shear stress-induced mechanotransduction protein deregulation and vasculopathy in a mouse model of progeria. Stem Cell Res Ther 2014, 5:41.

49. Bonello-Palot N, Simoncini S, Robert S, Bourgeois P, Sabatier F, Levy N, DignatGeorge F, Badens C: Prelamin A accumulation in endothelial cells induces premature senescence and functional impairment. Atherosclerosis 2014, 237:4552.

50. Osorio FG, Bárcena C, Soria-Valles C, Ramsay AJ, de Carlos F, Cobo J, Fueyo A, Freije JM, López-Otín C: Nuclear lamina defects cause ATM-dependent NFkappaB activation and link accelerated aging to a systemic inflammatory response. Genes Dev 2012, 26:2311-2324.

51. Benson EK, Lee SW, Aaronson SA: Role of progerin-induced telomere dysfunction in HGPS premature cellular senescence. J Cell Sci 2010, 123:26052612.

52. Datta S, Snow CJ, Paschal BM: A pathway linking oxidative stress and the Ran GTPase system in progeria. Mol Biol Cell 2014, 25:1202-1215.

* 53. Eisch V, Lu X, Gabriel D, Djabali K: Progerin impairs chromosome maintenance by depleting CENP-F from metaphase kinetochores in Hutchinson-Gilford progeria fibroblasts. Oncotarget 2016, 7:24700-24718.

This study links premature senescence and mitotic defects in HGPS cells. Progerin displaced CENP-F from kinetochores in metaphase, which caused increased chromatin lagging, binucleated cells and genomic instability, and delayed nuclear envelope reformation by trapping nuclear envelope proteins in the endoplasmic reticulum at the end of mitosis.

54. Arancio W, Pizzolanti G, Genovese SI, Pitrone M, Giordano C: Epigenetic involvement in Hutchinson-Gilford progeria syndrome: a mini-review. Gerontology 2014, 60:197-203. 
55. Loi M, Cenni V, Duchi S, Squarzoni S, López-Otín C, Foisner R, Lattanzi G, Capanni $\mathrm{C}$ : Barrier-to-autointegration factor (BAF) involvement in prelamin A-related chromatin organization changes. Oncotarget 2016, 7:15662-15677.

56. Prokocimer M, Barkan R, Gruenbaum Y: Hutchinson-Gilford progeria syndrome through the lens of transcription. Aging Cell 2013, 12:533-543.

** 57. Kubben N, Zhang W, Wang L, Voss TC, Yang J, Qu J, Liu GH, Misteli T: Repression of the antioxidant NRF2 pathway in premature aging. Cell 2016, 165:1361-1374.

This study demonstrates that NRF2 inactivation, by increasing chronic oxidative stress, is a key contributor to premature aging in HGPS. Suppressed NRF2 activity or oxidative stress recapitulated HGPS aging defects, and NRF2 reactivation in HGPS patient cells reversed progerin-associated nuclear aging defects and restored in vivo mesenchymal stem cell viability in an animal model.

58. Rivera-Torres J, Acín-Pérez R, Cabezas-Sánchez P, Osorio FG, González-Gómez C, Megías D, Cámara C, López-Otín C, Enríquez JA, Luque-García JL, et al.: Identification of mitochondrial dysfunction in Hutchinson-Gilford progeria syndrome through use of stable isotope labeling with amino acids in cell culture. J. Proteom. 2013, 91:466-477. 


\section{FIGURE LEGENDS}

FIGURE 1: DEFECTIVE PRELAMIN A PROCESSING IN HGPS: GENES AND PROTEINS. (A) In cells carrying the wild-type $L M N A$ sequence, normal splicing between exons 11 and 12 occurs and the precursor prelamin A is synthesized from LMNA mRNA. Through sequential post-translational modifications, prelamin $\mathrm{A}$ is rapidly processed to mature lamin A, which lacks the farnesylated and carboxymethylated prelamin A C-terminus. Inactivating mutations in the ZMPSTE24 gene cause the aberrant nuclear accumulation of permanently farnesylated and carboxymethylated prelamin A and accelerate aging. (B) Most HGPS patients carry in heterozygosis a de novo dominant synonymous mutation in the $L M N A$ gene (c.1824C $>$ T: GGC $>$ GGT; p.G608G). During pre-mRNA processing, this point mutation favors the use of a cryptic splice site in exon 11 , leading to the synthesis of progerin, a mutant form of prelamin A which lacks 50 aminoacid residues encompassing the ZMPSTE24 cleavage site. Like prelamin A, progerin retains the farnesyl and carboxymethyl modifications at the $\mathrm{C}$-terminus and accumulates in the nuclear envelope.

FIGURE 2: CELLULAR AND ORGANISMAL PHENOTYPIC CHANGES INDUCED BY PROGERIN. A-type lamins maintain nuclear architecture and regulate multiple cell functions, including higher-order chromatin organization, DNA replication and repair, signal transduction, gene transcription, and cell proliferation. Progerin expression in HGPS patients provokes, in a dominant-negative manner, cellular damage due to alterations in all lamin A-regulated functions (left). These cellular alterations cause organismal damage and accelerate aging (right). The cause of death in most HGPS patients is exacerbated cardiovascular disease, mainly through myocardial infarction or stroke.

FIGURE 3: MOLECULAR MECHANISMS DRIVING CALCIFICATION IN HGPS. Extracellular pyrophosphate (PPi) is a major inhibitor of VC mainly produced by vascular smooth muscle cells (VSMCs) from hydrolysis of ATP, another endogenous calcification inhibitor. Progerin accumulation in VSMCs impairs mitochondrial function, inhibiting intracellular ATP production. Extracellular ATP availability for PPi synthesis is reduced through increased activity of nucleotide pyrophosphatase/phosphodiesterase1 (eNTPD1). Extracellular PPi levels are further reduced by abnormally high tissuenonspecific alkaline phosphatase (TNAP) activity, which hydrolyzes PPi. Progeroid mice also have increased alkaline phosphatase (ALP) activity and reduced ATP and PPi levels in serum. Treatment of progeroid mice with PPi reduces aortic calcification, indicating that these changes in local and systemic polyphosphate metabolism contribute to calcium phosphate deposition in the vessel wall.

\section{FIGURE 4: PRELAMIN A ACCUMULATION IN VSMCS PROMOTES DNA DAMAGE, OSTEOGENIC DIFFERENTIATION AND CALCIFICATION.} Prelamin A accumulation in VSMCs activates the cell-cycle inhibitor p16 and the DNA damage response-related kinases ataxia-telangectasia-mutated (ATM) and ataxiatelangectasia Rad3 (ATR). This is accompanied by induced expression of osteogenic transcription factors, including osteocalcin, osteopontin, and osteoblast-specific Runrelated transcription factor-2 (RUNX2), which drive the osteogenic differentiation of VSMCs. This is followed by the secretion of pro-calcifying factors, including interleukin6 (IL-6), bone morphogenic protein 2 (BMP2) and osteoprotegerin, further promoting calcification. 

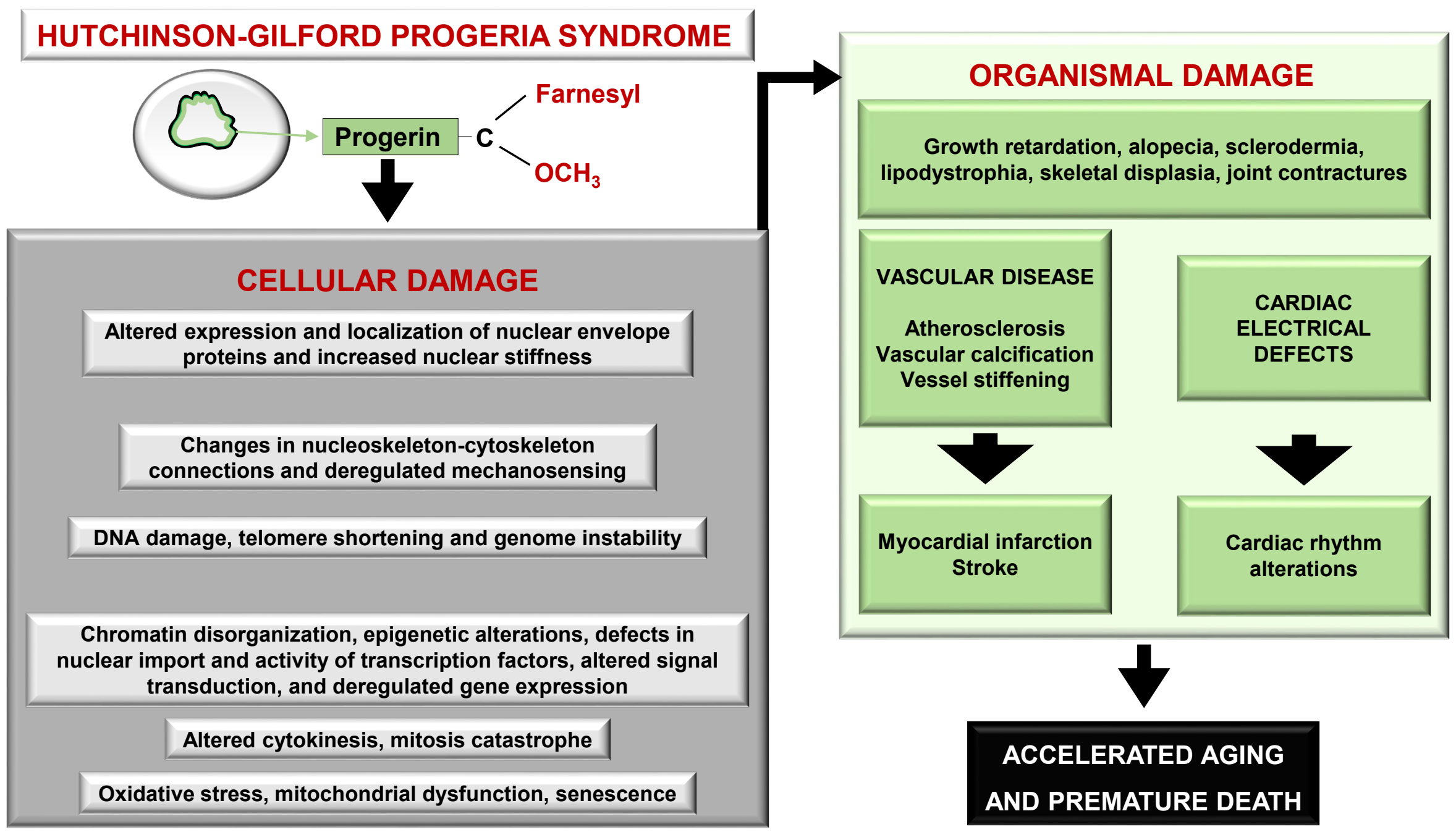


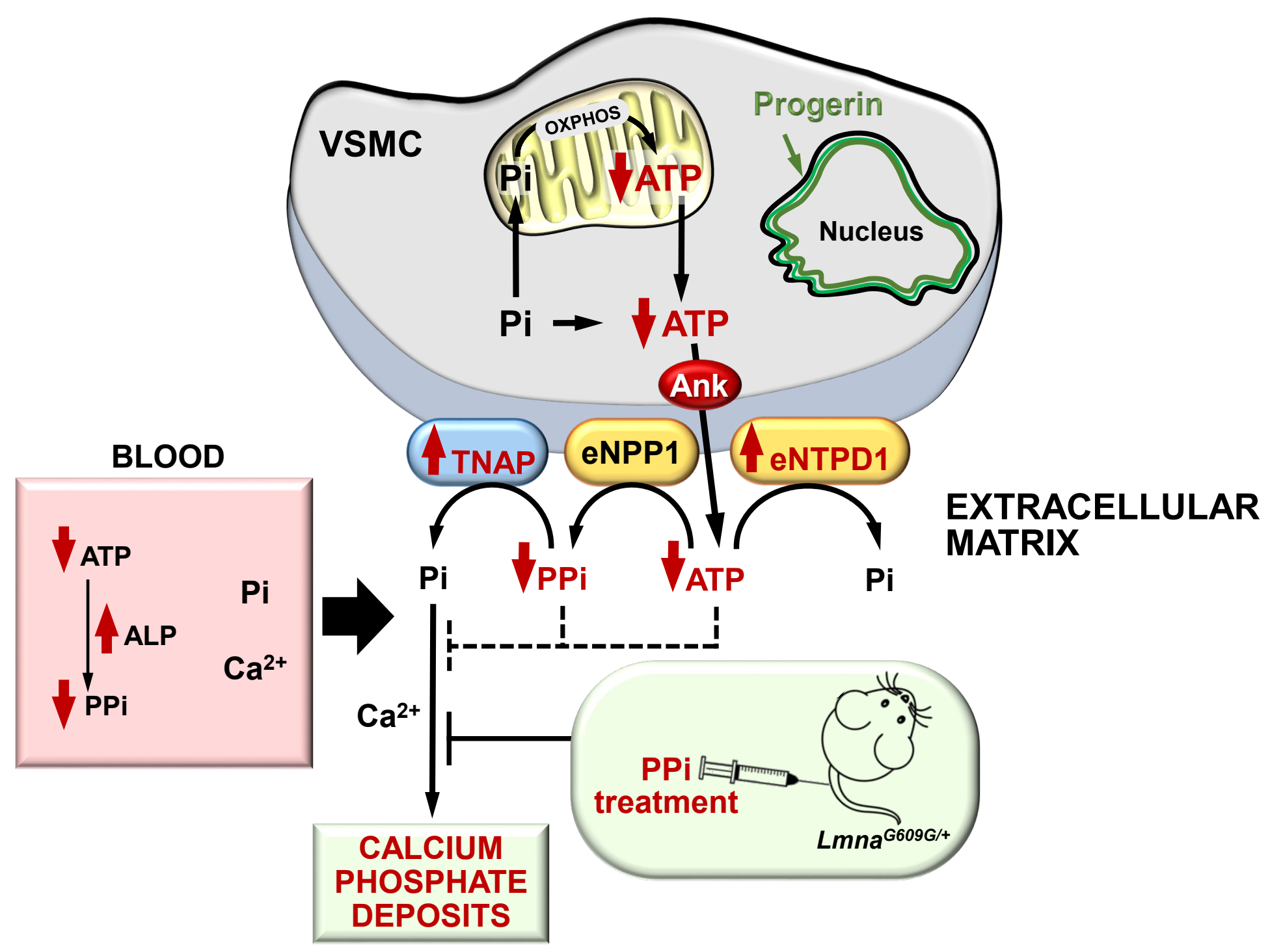




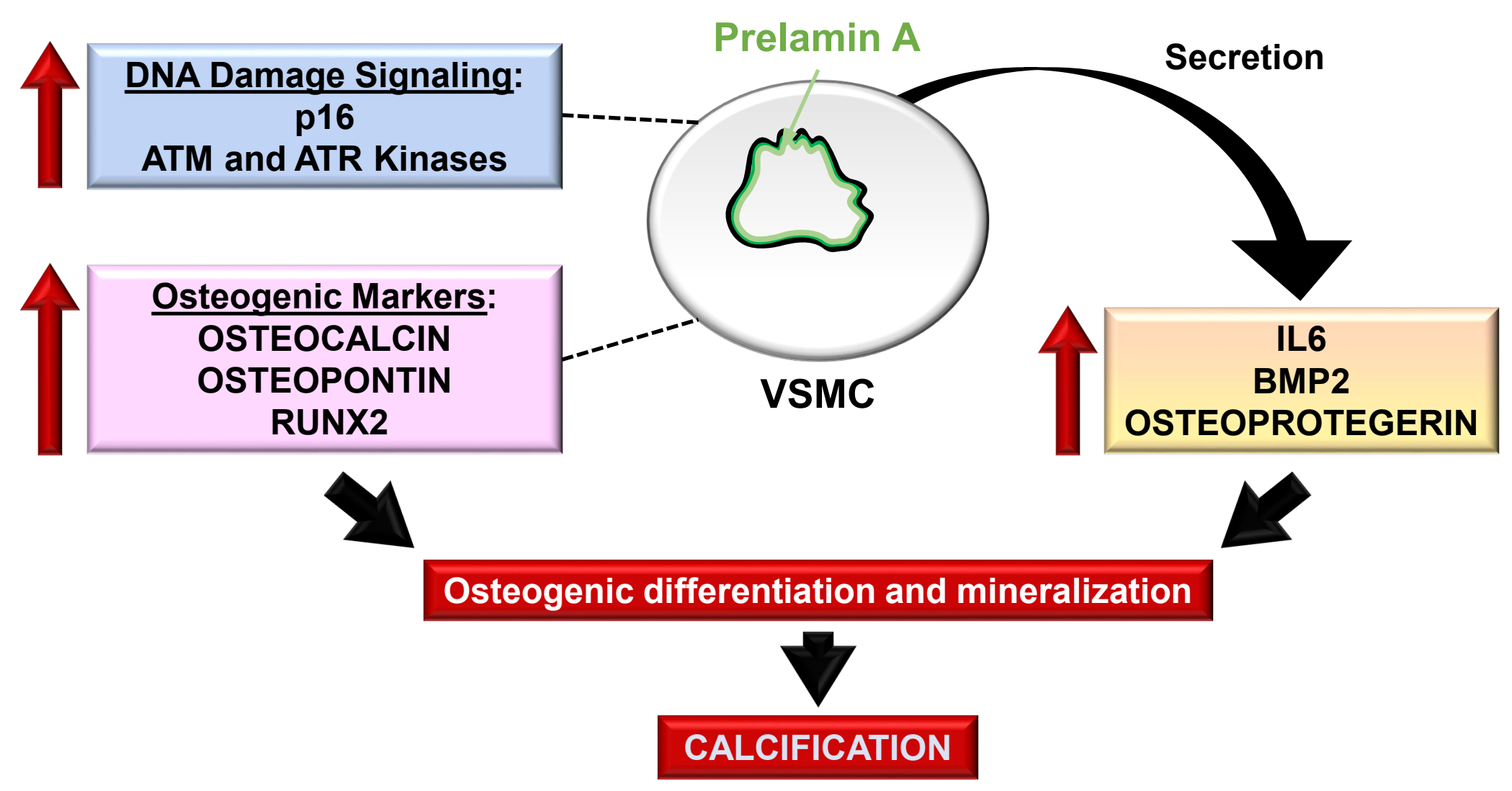

